\title{
Tea Consumption and Incidence of Type 2 Diabetes in Europe: The EPIC-InterAct Case-Cohort Study
}

\author{
The InterAct Consortium ${ }^{\uparrow *}$
}

\begin{abstract}
Background: In previous meta-analyses, tea consumption has been associated with lower incidence of type 2 diabetes. It is unclear, however, if tea is associated inversely over the entire range of intake. Therefore, we investigated the association between tea consumption and incidence of type 2 diabetes in a European population.

Methodology/Principal Findings: The EPIC-InterAct case-cohort study was conducted in 26 centers in 8 European countries and consists of a total of 12,403 incident type 2 diabetes cases and a stratified subcohort of 16,835 individuals from a total cohort of 340,234 participants with 3.99 million person-years of follow-up. Country-specific Hazard Ratios (HR) for incidence of type 2 diabetes were obtained after adjustment for lifestyle and dietary factors using a Cox regression adapted for a casecohort design. Subsequently, country-specific HR were combined using a random effects meta-analysis. Tea consumption was studied as categorical variable $(0,>0-<1,1-<4, \geq 4$ cups/day). The dose-response of the association was further explored by restricted cubic spline regression. Country specific medians of tea consumption ranged from 0 cups/day in Spain to $4 \mathrm{cups} /$ day in United Kingdom. Tea consumption was associated inversely with incidence of type 2 diabetes; the HR was $0.84[95 \% \mathrm{Cl} 0.71,1.00]$ when participants who drank $\geq 4$ cups of tea per day were compared with non-drinkers ( $p_{\text {linear }}$ trend $=0.04)$. Incidence of type 2 diabetes already tended to be lower with tea consumption of $1-<4$ cups/day (HR=0.93 $[95 \% \mathrm{Cl} 0.81,1.05])$. Spline regression did not suggest a non-linear association $\left(p_{\text {non-linearity }}=0.20\right)$.
\end{abstract}

Conclusions/Significance: A linear inverse association was observed between tea consumption and incidence of type 2 diabetes. People who drink at least 4 cups of tea per day may have a 16\% lower risk of developing type 2 diabetes than nontea drinkers.

Citation: The InterAct Consortium (2012) Tea Consumption and Incidence of Type 2 Diabetes in Europe: The EPIC-InterAct Case-Cohort Study. PLoS ONE 7(5): e36910. doi:10.1371/journal.pone.0036910

Editor: Christian Herder, German Diabetes Center - Leibniz Center for Diabetes Research at Heinrich Heine University Duesseldorf, Germany

Received October 31, 2011; Accepted April 10, 2012; Published May 30, 2012

Copyright: ( 2012 van Woudenbergh, The InterAct Consortium. This is an open-access article distributed under the terms of the Creative Commons Attribution License, which permits unrestricted use, distribution, and reproduction in any medium, provided the original author and source are credited.

Funding: The EPIC-InterAct project was funded by the European Union (Integrated Project LSHM-CT-2006-037197 in the Framework Program 6 of the European Community). In addition, EPIC-InterAct investigators acknowledge funding from the following agencies:-DLvdA, AMWS, and HBBdM: Dutch Ministry of Public Health, Welfare and Sports (VWS), Netherlands Cancer Registry (NKR), LK Research Funds, Dutch Prevention Funds, Dutch ZON (Zorg Onderzoek Nederland), World Cancer Research Fund (WCRF), Statistics Netherlands (The Netherlands); EA and AB: Health Research Fund (FIS), Regional Governments of Andalucía, Basque Country, Murcia and Navarra, ISCIII RETIC (RD06/0020) (Spain); JWJB and IS: Verification of diabetes cases was additionally funded by NL Agency grant IGE05012 and an Incentive Grant from the Board of the UMC Utrecht; AM: Compagnia di San Paolo; MDC: Health Research Fund (FIS) of the Spanish Ministry of Health; Murcia Regional Government ( ${ }^{\circ}$ 6236), and CIBER Epidemiología y Salud Pública (CIBERESP), Spain; FC: Cancer Research UK; AT, JH, CCD, and KO: Danish Cancer Society; OR: Västerbotten County Council; PWF: Swedish Research Council, Novo Nordisk, Swedish Diabetes Association, Swedish Heart-Lung Foundation; JRQ: Regional Government of the Principality of Asturias (Spain); PN and LG: Swedish Research Council; RT: AIRE-ONLUS Ragusa, AVIS-Ragusa, Sicilian Regional Government; KTK: Medical Research Council UK, Cancer Research UK. The funders had no role in study design, data collection and analysis, decision to publish, or preparation of the manuscript.

Competing Interests: Paul W. Frank received funding from a commercial source (Novo Nordisk). This does not alter the authors' adherence to all the PLoS ONE policies on sharing data and materials. All other authors have declared that no competing interests exist.

* E-mail: truus.vanwoudenbergh@wur.nl

- Membership of the InterAct Consortium is provided in the Acknowledgments.

\section{Introduction}

Increasing our understanding of modifiable lifestyle factors associated with the development of type 2 diabetes is important, as the prevalence of diabetes is increasing rapidly [1]. Obesity is a major risk factor for the development of type 2 diabetes [2], but dietary factors may also play a role. One dietary factor of interest is tea consumption. Tea consumption may lower the risk of type 2 diabetes by influencing glucose digestion, glucose uptake, and by protecting beta-cells from free-radical damage [3-5]. This beneficial effect may be due to the polyphenols present in tea.

A meta-analysis, including nine cohort studies, reported that drinking at least 4 cups of tea per day was associated with a $20 \%$ lower risk, whereas drinking $>0-<1$ or $1-3$ cups per day did not lower the risk of diabetes compared with non-tea drinkers [6]. In line with this, no association was observed when tea consumption was studied as continuous variable. This may indicate that the protective effect of tea is restricted to people with a high tea consumption, although a potential biological mechanism has not yet been described.

Studies in which tea consumption is low, therefore, may not observe an association between tea consumption and risk of type 2 diabetes. This is supported by three out of the four additional cohort studies, which were published after the meta-analysis [710]. Two of those studies, in which the highest tea consumption category was relatively low, at least 3 cups per day [7] or at least 2 
cups per day [8], did not observe an association between tea consumption and risk of diabetes, whereas one study, in which the highest tea consumption category was more than 5 cups per day, observed a substantial lower risk [9]. In contrast, one study did not observe an association, even though the highest category of tea consumption included participants who drank at least 4 cups per day [10]. So, to date it is unclear whether or how tea consumption is associated with risk of type 2 diabetes.

Therefore, we investigated the association between tea consumption and incidence of type 2 diabetes in European citizens who were part of the EPIC-InterAct project. The size of the study is comparable with the meta-analyses reported to date on this topic $[6,11]$ and provides the opportunity to explore a potential nonlinear association between tea consumption and risk of type 2 diabetes across European countries.

\section{Methods}

\section{Ethics statement}

The study complied with the Declaration of Helsinki. The Internal Review Board of the International Agency for Research on Cancer and the Institutional Review Board of all centers, i.e., France, Heidelberg, Postdam, Copenhagen, Aarhus, Asturias, Granada, San Sebastian, Murcia, Navarro, Cambridge, Oxford, Imperial, Florence, Milan, Ragusa, Turin, Naples, Bilthoven, Utrecht, Malmö, and Umeä, approved the EPIC study. Written consent was obtained from each EPIC participant at enrolment into the study.

\section{Population}

The EPIC-InterAct project is a case-cohort study embedded in the EPIC study. The EPIC study is a prospective study conducted in 10 European countries [12]. Eight countries also participated in the EPIC-InterAct project (Spain, Italy, Sweden, France, Denmark, Germany, The Netherlands, and United Kingdom), with a total of 26 centers. The rationale and design of the EPIC-InterAct project has been described in detail elsewhere [13]. In short, a center-stratified random sample of 16,835 participants, aged 2079 years, was taken as sub-cohort. Subsequently, a number of 548 participants with prevalent diabetes and 133 with unknown diabetes status were excluded, resulting in 16,154 participants. After verification of all eligible EPIC participants for diabetes incidence, 12,403 verified cases were obtained of which 778 belongs to the sub-cohort.

Our analysis included 26,039 participants of the 27,779 participants included in the EPIC-InterAct-project, because we excluded in consecutive order 117 participants without dietary data, 619 with unreliable food intake data (top and bottom $1 \%$ of the distribution of energy intake to basal energy requirement assessed by WHO/FAO/UNU equation including weight and height) [14], and 955 with missing information about potential confounders which were included in the final model (289 without physical activity measurements, 134 without information on smoking status, 367 without information on educational status, and 165 without information on body mass index (BMI)). Furthermore, since few participants from Spain $(n=39)$ and Italy $(n=10)$ drank $\geq 4$ cups of tea per day, country-specific hazard ratios (HR) comparing $\geq 4$ vs. 0 cups per day could not be obtained in Spain and Italy. Therefore, those 49 participants were also excluded.

\section{Dietary intake including tea consumption}

Dietary intake over the last 12 months was assessed by countryspecific or center-specific semi-quantitative or quantitative dietary questionnaires, validated within each country [12]. More information about the questionnaires can be found elsewhere [12]. All questionnaires included at least one question about tea consumption. Each center converted the information about tea consumption into grams per day. For the analysis, tea consumption in grams per day was divided by 125 to be able to calculate HR by cups per day. In line with the meta-analysis by Jing et al. [6], the frequency of tea consumption was divided into 4 categories: $0,>0$ $<1,1-<4, \geq 4$ cups per day.

\section{Diabetes incidence}

A pragmatic, high sensitivity approach for case ascertainment was used in order to identify all potential incident type 2 diabetes cases and excluding all individuals with prevalent diabetes [13]. Briefly, ascertainment of incident diabetes involved a review of the existing EPIC datasets at each center using multiple sources of evidence including self-report, hospital admissions, linkage to primary care registers, linkage to secondary care registers, linkage to drug registers, and mortality data. Cases in Denmark and Sweden were not ascertained by self-report, but via diabetes and pharmaceutical registers. Hence, all ascertained cases were considered to be verified. To increase the ability to exclude false negatives for countries other than those from Denmark and Sweden, we sought further evidence for all cases with information on incident type 2 diabetes from less than 2 independent sources which have been described in detail elsewhere [13]. Follow-up was censored at the date of diagnosis, the 31 st of December 2007, or the date of death, whichever occurred first.

\section{Non-dietary covariates}

Socio-demographic and lifestyle information, e.g., age, sex, education level, smoking status, and physical activity during work and leisure time [15], was obtained with questionnaires at baseline.

Questionnaires were also used to obtain information about diseases of the participant and his family, i.e., history of angina pectoris (not in The Netherlands, Sweden, and one center in Germany), history of myocardial infarction, history of stroke (not in one center in Sweden), presence and/or treatment for hypertension, presence and/or treatment of hyperlipidemia (not in one center in Sweden), and family history of type 2 diabetes (not in Spain, Italy, one center in Germany, and one center in the United Kingdom).

Information about height and weight was obtained using a standard protocol during a visit at the research center at baseline for all participants, except in France and in some of the participants from one center in the United Kingdom. Selfreported or corrected height and weight were used in those centers without measured height and weight [12].

\section{Statistical analysis}

The association between tea consumption $(0,>0-<1,1-<4, \geq 4$ cups/day) and risk of type 2 diabetes was examined by country using modified Cox proportional hazard models with age as underlying time scale. The models were modified for the casecohort design according to the Prentice method [16]. In order to adjust for time to follow-up, age at recruitment (1-year categories) was included as stratum variable. Summary $\mathrm{HR}$ and $95 \%$ confidence intervals $(95 \% \mathrm{CI})$ were obtained by pooling countryspecific HR using random effects meta-analyses and visualized in forest plots. Between country heterogeneity was assessed by $I^{2}$ statistic, i.e., the percentage of variation in the HR attributable to between country heterogeneity [17]. 
To obtain adjusted country-specific HR, four Cox models were constructed. Variables included in these models were considered main potential confounders in the association between tea consumption and risk of type 2 diabetes based upon literature. Model 1 included, in addition to tea consumption, four other covariates: sex, smoking status (never, former, current), physical activity level (inactive, moderately inactive, moderately active, and active), and education level (lowest, secondary, and highest). Model 2 was similar to model 1 with additional adjustments for energy and intake of seven nutrients: protein (energy-\%), carbohydrates (energy-\%), saturated fatty acids (energy-\%), mono-unsaturated fatty acids (energy-\%), poly-unsaturated fatty acids (energy-\%), alcohol $(0,>0-6,>6-12,>12-24$, and $>24 \mathrm{~g} /$ $\mathrm{d})$, and fiber $(\mathrm{g} / \mathrm{d})$. Model 3 was similar to model 2 with additional adjustments for intake of drinks: coffee $(\mathrm{g} / \mathrm{d})$, juices $(\mathrm{g} / \mathrm{d})$, softdrinks (g/d), and milk (g/d). Model 4 was similar to model 3 with additional adjustment for BMI $\left(\mathrm{kg} / \mathrm{m}^{2}\right)$.

After investigating the association between tea consumption as a categorical variable and risk of type 2 diabetes, the dose-response of the association was further explored by studying linear trends across categories, by restricted cubic spline regression, and by studying consumption of tea (cups per day) as a continuous variable. To test for linear trends across categories, the median value of each category of tea consumption was modeled as a continuous variable. The restricted cubic spline regression was performed using SAS Macro RCS, which was also based on the modified Cox proportional hazard regression. The knots were located at 1, 4, 7 cups per day and non-tea drinkers were used as reference group. This analysis was adjusted as described for model 4.

Potential effect modification was investigated by including in the models an interaction term between tea categories and sex or tea categories and BMI categories and by studying the association between tea consumption and risk of type 2 diabetes by sex (men: $n=11,249$; women: $n=15,030$ ) and by BMI (Normal: $\mathrm{BMI}<25.0 \mathrm{~kg} / \mathrm{m}^{2}: \quad n=8,267$; $\quad$ Overweight: $\mathrm{BMI} \geq 25.0-$ $<30.0 \mathrm{~kg} / \mathrm{m}^{2}: n=10,840$; Obese: BMI $\left.\geq 30.0 \mathrm{~kg} / \mathrm{m}^{2}: n=6,932\right)$.

To investigate the robustness of the associations, sensitivity analyses were performed by excluding one by one participants for each of the following diseases at baseline: a history of stroke $(n=261)$, a history of angina pectoris $(n=521)$, a history of a myocardial infarction $(n=545)$, hypertension $(n=5,682)$, and hyperlipidemia $(n=4,362)$. Furthermore, participants with a family history of diabetes $(n=2,928)$ and who developed type 2 diabetes within 2 years $(n=955)$ were excluded in the sensitivity analyses. We excluded these participants in the sensitivity analyses, because they may have changed recently their diet. In centers which did not obtain information about disease history, participants were considered as not having the disease at baseline.

Analyses were carried out using the statistical software program SAS version 9.1, except for the random effects meta-analyses which were conducted in STATA 11.0. A two-sided p-value $\leq 0.05$ was considered as statistically significant for all analyses.

\section{Results}

Overall, 64\% of this European study population reported that they drank tea. The highest median total tea consumption was observed in the United Kingdom (3.8 (IQR 3.8-6.8) cups per day); the lowest in Spain (0.0 (IQR 0-0) cups per day) (Figure 1). The median total tea consumption among drinkers was 1.2 (IQR 0.33.7) cups per day. In general, participants with higher tea consumption had a lower BMI, had a higher level of education, and smoked less (Table 1). Intake of carbohydrate and saturated fatty acids was higher, whereas intake of mono-saturated fatty acids was lower across tea categories. Tea drinkers drank less alcohol, but more coffee, soft drinks, and juices than non-tea drinkers.

When tea consumption was divided into categories and countryspecific HR were combined, the crude analysis showed that, for all categories, participants who drank tea had a lower risk of developing type 2 diabetes compared with non-tea drinkers (Table 2). The risk estimates for type 2 diabetes in all categories of tea consumption were attenuated slightly in model 1. Additional adjustment for the intake of nutrients (model 2) and drinks (model 3) did not affect the risk estimates. Adjustment for BMI (model 4), however, attenuated the risk estimates further, but risk of type 2 diabetes was still $16 \%$ lower in participants drinking at least 4 cups of tea per day compared with non-tea drinkers $\left(\mathrm{HR}_{\geq 4} \mathrm{cups} / \mathrm{d}\right.$ vs. ${ }_{0}=0.84$ [95\%CI 0.71, 1.00]; $p_{\text {linear trend }}=0.04$ ). Risk of type 2 diabetes already tended to be lower among participants drinking 1 to 4 cups per day compared with non-tea drinkers (Model $4 \mathrm{HR}_{1-}$ $<4 \mathrm{cups} / \mathrm{d}$ vs. $0=0.93$ [95\% CI 0.81, 1.05]) (Table 2; Figure 2). No evidence of between country heterogeneity was observed in any category of tea consumption (Figure 2).

The association was further explored by performing spline regression and by studying tea consumption as continuous variable. Cubic spline regression confirmed that risk of type 2 diabetes may be lower with higher intake of tea, although it suggests that the risk may level off after 5 cups per day ( $p_{\text {non- }}$ linearity $=0.20$; Figure 3). Since the risk reduction may level off, the association between tea consumption on a continuous scale and risk of type 2 diabetes was restricted to participants drinking 5 cups per day or less $(n=23,778)$. This analysis suggested that 1 cup of tea per day was associated with a $3.1 \%$ lower risk, which was nearly statistically significant (Model 4: HR 0.97 [95\%CI 0.94, $1.00], p=0.06)$.

Stratified analyses showed that effect modification was not observed for sex (Model 4: $p_{\text {interaction }}=0.14: M e n: \mathrm{HR}_{\geq 4} \mathrm{cups} / \mathrm{d}$ vs. ${ }_{0}=0.89[95 \%$ CI $0.71,1.10] ;$ Women: $\mathrm{HR}_{\geq 4} \mathrm{cups} / \mathrm{d}$ vs. $0=0.82$ $[95 \%$ CI $0.58,1.16]$ ) and for BMI (Model 4: $p_{\text {interaction }}=0.26$ : $B M I<25.0: \quad \mathrm{HR}_{\geq 4} \quad \mathrm{cups} / \mathrm{d} \quad$ vs. $0=0.79 \quad[95 \% \mathrm{CI} \quad 0.52,1.19]$; $B M I \geq 25.0-<30.0: \mathrm{HR}_{\geq 4}$ cups $/$ d vs. $0=0.87$ [95\%CI 0.63, 1.20]; $B M I \geq 30.0: \mathrm{HR}_{\geq 4}$ cups/d vs. $0=0.79$ [95\%CI 0.59, 1.05]).

None of the sensitivity analyses changed the results substantially (data not shown).

\section{Discussion}

In this large European population, a linear inverse association was observed between tea consumption and incidence of type 2 diabetes. This significant linear association and the spline regression suggests that a threshold of drinking at least 4 cups of tea per day to lower risk of type 2 diabetes does not appear to exist.

Strengths of our analyses included the ability to study the association between tea consumption and risk of type 2 diabetes in populations from eight European countries, resulting in a larger variation of tea consumption than when these countries were analyzed separately. Our results were also strengthened by the standardized protocol to verify cases of diabetes among countries and to process information about lifestyle and dietary factors. As such, consumption of tea was converted into grams per day for all countries, through which we were able to standardize cup size among countries in the analysis.

Our observation that drinking tea was associated inversely with risk of type 2 diabetes, was in line with the observation of two published meta-analyses equaling the power of our study $[6,11]$. We observed a protective association on risk of type 2 diabetes 


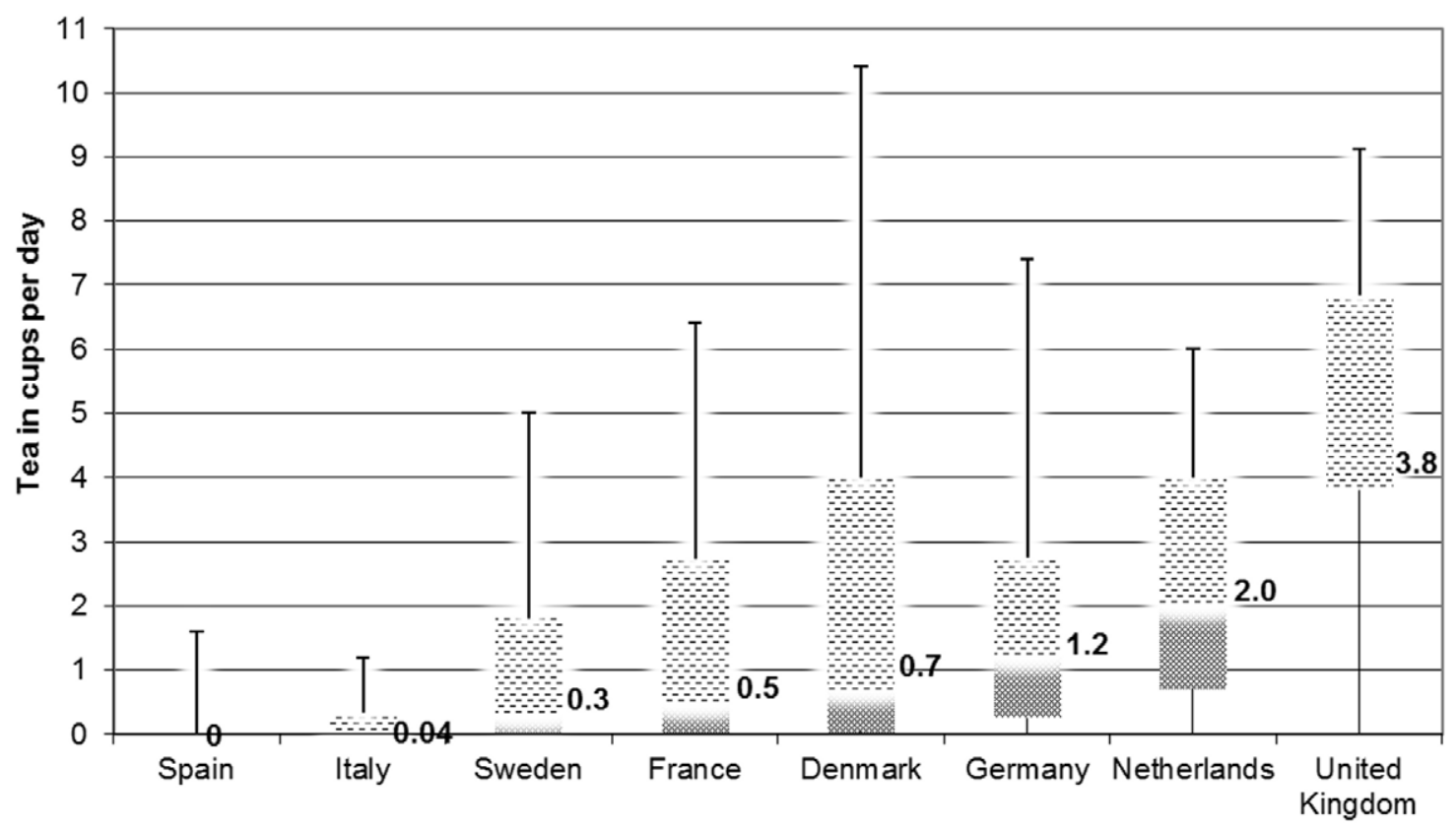

Figure 1. Tea consumption based on data from a food frequency questionnaire in the sub-cohort of the EPIC-InterAct project by country $(n=\mathbf{1 5 , 2 2 7})$. Bar represents median (p25-p75); error line represents $p 5$ till p95. doi:10.1371/journal.pone.0036910.g001

with habitual tea consumption of at least 4 cups per day, Jing et al. [6] also with at least 4 cups per day, and Huxley et al. [11] with at least 3-4 cups per day. In line with the meta-analysis by Jing et al., we observed that tea drinkers who drank $<1$ cups per day had a similar risk of type 2 diabetes as non-tea drinkers. Risk of type 2 diabetes, however, already tended to be lower with $1-<4$ cups of tea per day in our analysis. Together with the results of the spline regression, our results, therefore, do not support that the protective effect of tea consumption is restricted to participants reporting the highest intake of tea. Therefore, even lower doses may reduce risk of type 2 diabetes.

Our spline regression suggests that the risk reduction leveled off at around 5 cups per day. Potential mechanisms, however, explaining the observed plateau around 5 cups per day are not established. Furthermore, we could not study country-specific associations between a very high tea consumption $(>7$ cups per day) and risk of type 2 diabetes in a sufficient number of people in our analysis. We already had to exclude Spain and Italy from the highest tea category, because of small numbers in this category. The biological mechanism underlying a beneficial effect of tea, however, is unlikely to differ between Northern and Southern European countries.

The flavonoids present in tea are of importance, because the beneficial effect of tea on risk of type 2 diabetes may be attributable to these components [4,5]. Catechins, theaflavins, and thearubigins are the most prominent flavonoids in tea. These flavonoids, predominately epigallocatechin gallate (EGCG), have been shown to slow down carbohydrate digestion, to inhibit carbohydrate absorption by competitively binding with the sodium-glucose transporter-1 (SGLT-1), to increase glucose uptake in muscle and fat cells by changes in GLUT-4 expression, to enhance insulin secretion, and to protect beta-cells from freeradical damage [4,5]. All these pathways can affect glucose concentrations and, thereby, could explain a beneficial effect of tea consumption on risk of type 2 diabetes. Most randomized controlled trials investigating the association between long-term tea consumption and markers of glucose of insulin metabolism, however, showed no associations [18-23].

Studies investigating the association between tea consumption and risk of type 2 diabetes may differ by several factors, such as the type of tea consumed, the preparation method used, the cup size used, and the sample size under investigation. The main type of tea consumed may result in discrepancies among studies and among countries in our study, because types of tea differ in chemical composition. Herbal teas may contain less anti-oxidants than black and green tea, because herbal teas, in contrast to black or green tea, are not derived from the Camellia Sinensis plant [24]. The difference in anti-oxidant capacity may indicate that the beneficial effect of drinking tea is stronger for black or green tea than for herbal tea. It is likely that tea consumption in our study mainly reflects the intake of black tea, because at the time of dietary data collection in this study, green tea and herbal tea were not as popular as black tea in most countries. Unfortunately, we could not investigate whether the type of tea consumed affects risk of type 2 diabetes differently, because detailed information about type of tea was not collected in most countries.

The preparation method used may also result in discrepancies. The preparation method, including brewing time and substances added, can influence the amount of flavonoids present in a cup of tea $[25,26]$ and, consequently, the association between tea consumption and risk of type 2 diabetes. Studies, therefore, might find an association at $1-<4$ cups of tea per day if the brewing time is long in general, whereas other studies might not find an association if the brewing time is short. Since information about brewing time was lacking in all published studies, including ours, it was not possible to adjust for brewing time. As brewing time was not reported in our study, non-differential misclassification of actual amount of tea consumption could have occurred within countries. Next to a short brewing time, adding milk may also lower the bioavailability of flavonoids due to the interaction between milk proteins and flavonoids present in tea [27]. Five out of six trials, however, showed that adding milk to tea did not affect 
Table 1. Characteristics of the sub-cohort of the EPIC-InterAct project by categories of tea consumption $(n=15,227)$.

\begin{tabular}{|c|c|c|c|c|}
\hline & \multirow[t]{2}{*}{ None } & \multirow{2}{*}{$\frac{>0-<1}{\text { (cups/day) }}$} & \multirow{2}{*}{$\frac{1-<4}{\text { (cups/day) }}$} & \multirow{2}{*}{$\begin{array}{l}\geq 4 \\
\text { (cups/day) }\end{array}$} \\
\hline & & & & \\
\hline & $(n=5,458)$ & $(n=4,032)$ & $(n=3,444)$ & $(n=2,293)$ \\
\hline Age (years) & $51.7(8.6)$ & $51.4(9.1)$ & $53.0(9.7)$ & $54.7(8.8)$ \\
\hline Body mass index $\left(\mathrm{kg} / \mathrm{m}^{2}\right)$ & $27.0(4.3)$ & $25.8(4.0)$ & $25.5(4.0)$ & $25.0(3.8)$ \\
\hline Male (\%) & 40.5 & 41.3 & 32.7 & 33.6 \\
\hline \multicolumn{5}{|l|}{ Country (\%) } \\
\hline Spain & 83.0 & 7.5 & 9.5 & 0 \\
\hline Italy & 43.1 & 44.2 & 12.8 & 0 \\
\hline Sweden & 33.6 & 30.5 & 28.2 & 7.7 \\
\hline France & 36.7 & 21.1 & 27.4 & 15.1 \\
\hline Denmark & 17.3 & 38.8 & 9.8 & 34.1 \\
\hline Germany & 6.1 & 39.6 & 36.5 & 17.8 \\
\hline Netherlands & 8.3 & 18.7 & 42.1 & 31.0 \\
\hline United Kingdom & 5.2 & 8.9 & 39.0 & 46.9 \\
\hline Smoking (\% current) & 31.9 & 27.5 & 19.6 & 18.8 \\
\hline Education level (\% high) & 13.7 & 21.7 & 25.3 & 28.8 \\
\hline Physical activity (\% inactive) & 31.6 & 20.1 & 20.4 & 15.6 \\
\hline Hypertension (\%) & 17.5 & 19.1 & 20.3 & 17.4 \\
\hline Hyperlipidaemia (\%) ${ }^{1}$ & 17.9 & 15.8 & 17.0 & 10.3 \\
\hline Family history of diabetes $(\%)^{2}$ & 13.9 & 17.1 & 16.9 & 17.9 \\
\hline Stroke $(\%)^{3}$ & 0.7 & 0.9 & 1.1 & 0.7 \\
\hline Angina Pectoris $(\%)^{4}$ & 1.3 & 2.4 & 3.1 & 2.4 \\
\hline Myocardial infarct (\%) & 1.1 & 1.4 & 1.6 & 1.7 \\
\hline \multicolumn{5}{|l|}{ Dietary intake } \\
\hline Total energy $(\mathrm{kcal} / \mathrm{d})$ & $2188(662)$ & $2140(645)$ & $2067(591)$ & $2124(603)$ \\
\hline Protein (energy\%) & $18.0(3.2)$ & $16.4(2.8)$ & $16.5(3.1)$ & $16.6(2.9)$ \\
\hline Fat (energy\%) & $35.4(6.1)$ & $34.6(5.7)$ & $34.6(5.8)$ & $34.0(5.6)$ \\
\hline Saturated fatty acids & $12.4(3.6)$ & $13.6(3.3)$ & $13.7(3.3)$ & $13.8(3.1)$ \\
\hline Mono-unsaturated fatty acids & $14.6(3.7)$ & $13.0(3.1)$ & $12.3(3.0)$ & $11.3(2.2)$ \\
\hline Poly-unsaturated fatty acids & $5.6(2.1)$ & $5.2(1.6)$ & $5.8(1.8)$ & $5.9(1.8)$ \\
\hline Carbohydrate (energy\%) & $42.2(7.2)$ & $44.7(6.9)$ & $45.1(6.8)$ & $45.5(6.7)$ \\
\hline Fiber $(\mathrm{g} / \mathrm{d})$ & $22.7(8.1)$ & $22.1(7.4)$ & $22.4(7.2)$ & $24.6(8.0)$ \\
\hline Alcohol (\%>24 g/d) & 22.6 & 19.9 & 15.1 & 15.5 \\
\hline Coffee $(g / d)$ & $154(60-400)$ & $400(130-700)$ & $363(125-525)$ & $375(86-500)$ \\
\hline Soft drinks (g/d) & $0(0-29)$ & $16(0-86)$ & $14(0-90)$ & $16(0-90)$ \\
\hline Juices $(\mathrm{g} / \mathrm{d})$ & $1(0-25)$ & $22(3-94)$ & $40(4-120)$ & $29(3-100)$ \\
\hline Milk (g/d) & $180(61-300)$ & $138(25-271)$ & $150(25-295)$ & $193(36-387)$ \\
\hline
\end{tabular}

Values are expressed as Mean (Standard Deviation), Median (p25-p75), or percentage.

${ }^{1}$ Based on $n=13,345$, because information about hyperlipidemia was not collected in one center of Sweden.

${ }^{2}$ Based on $n=8,802$, because information about family history of diabetes was not collected in Italy, Spain, one center of Germany, and one center of the United

Kingdom.

${ }^{3}$ Based on $n=14,262$, because information about a history of stroke was not collected in one center of Sweden.

${ }^{4}$ Based on $n=10,168$, because information about a history of angina was not collected in Sweden, The Netherlands, and one center of Germany.

doi:10.1371/journal.pone.0036910.t001

the bioavailability of tea flavonoids or anti-oxidant capacity after consumption [26,28-32]. Furthermore, the beneficial effect of tea consumption on risk of type 2 diabetes may be counterbalanced by the addition of sugar. Since we did not observe heterogeneity among countries in our study, we do not think that differences in preparation methods among countries have influenced our results.

In our study, tea consumption was associated with a healthier lifestyle, e.g., people who drank tea were more physically active and smoked less. This may indicate that the inverse association between tea consumption and risk of type 2 diabetes reflects a healthier lifestyle rather than tea consumption itself. In our analysis, however, we tried to disentangle the effect of a healthier lifestyle from tea consumption by adjusting the HR for a range of lifestyle and dietary factors, and by excluding people with chronic diseases at baseline in sensitivity analyses. Inclusion of lifestyle and dietary factors apart from BMI, however, did not change the risk 


\section{Country}

$<1$ vs. 0 cupsid

Spain

Italy

Sweden

France

Denmark

Germany

Netherlands

United Kingdom

Subtotal (l-squared $=34.9 \%, p=0.150$ )

$1-<4$ vs. 0 cups/d

Spain

Italy

Sweden

France

Denmark

Germany

Netherlands

United Kingdom

Subtotal (l-squared $=31.3 \%, p=0.178$ )

4 or morevs. 0 cups/d

Sweden

France

Denmark

Germany

Netherlands

United Kingdom

Subtotal ( $(-$ squared $=21.0 \%, p=0.276$ )

NOTE: Weights are from random effects analysis
$\mathrm{HR}(95 \% \mathrm{Cl})$

$1.27(1.01,1.59)$

$0.94(0.78,1.14)$

$1.18(0.99,1.42)$

$1.18(0.75,1.86)$

$0.92(0.75,1.13)$

$1.02(0.71,1.46)$

$0.72(0.46,1.12)$

$0.79(0.42,1.50)$

$1.03(0.91,1.16)$

$1.05(0.84,1.30)$

$0.78(0.57,1.07)$

$0.98(0.82,1.17)$

$0.79(0.50,1.24)$

$1.21(0.91,1.61)$

$0.94(0.65,1.36)$

$0.66(0.44,0.99)$

$0.70(0.43,1.15)$

$0.93(0.81,1.05)$

$1.02(0.74,1.41)$

$0.70(0.41,1.19)$

$0.95(0.75,1.21)$

$0.89(0.59,1.35)$

$0.57(0.36,0.89)$

$0.70(0.42,1.16)$

$0.84(0.71,1.00)$

Figure 2. Association between tea consumption as a categorical variable $(>0-<1$ vs. $0,1-<4$ vs. $0, \geq 4$ vs. 0 cups/d) based on data from a food frequency questionnaire and risk of type $\mathbf{2}$ diabetes $(\boldsymbol{n}=\mathbf{2 6 , 0 3 9})$. Country-specific Hazard Ratios (HR) and $95 \%$ Confidence Intervals $(95 \% \mathrm{Cl})$ were pooled using random effects meta-analyses. HR were adjusted for sex, smoking status, physical activity level, education level, intake of energy, protein, carbohydrates, saturated fatty acids, mono-unsaturated fatty acids, poly-unsaturated fatty acids, alcohol, fiber, coffee, juices, soft-drinks, milk, and body mass index. doi:10.1371/journal.pone.0036910.g002

Table 2. Hazard Ratios (HR) and $95 \%$ confidence intervals $(95 \% \mathrm{Cl})$ for incident type 2 diabetes by categories of tea consumption in cups per day $(n=26,039)$.

\begin{tabular}{|c|c|c|c|c|c|c|c|c|c|c|c|c|c|}
\hline & \multirow[b]{2}{*}{$\mathrm{N}$ total } & \multirow[b]{2}{*}{ Cases } & \multirow[b]{2}{*}{ Median } & \multicolumn{2}{|c|}{ Crude } & \multicolumn{2}{|c|}{ Model 1} & \multicolumn{2}{|c|}{ Model 2} & \multicolumn{2}{|c|}{ Model 3} & \multicolumn{2}{|c|}{ Model 4} \\
\hline & & & & HR & (95\% Cl) & HR & $(95 \% \mathrm{Cl})$ & HR & $(95 \% \mathrm{Cl})$ & HR & $(95 \% \mathrm{Cl})$ & HR & $(95 \% \mathrm{Cl})$ \\
\hline 0 & 9,499 & 4,389 & 0 & 1 & & 1 & & 1 & & 1 & & 1 & \\
\hline$>0-<1$ & 7,060 & 3,197 & 0.23 & 0.89 & $(0.80,0.99)$ & 0.93 & $(0.81,1.07)$ & 0.96 & $(0.84,1.10)$ & 0.97 & $(0.85,1.10)$ & 1.03 & $(0.91,1.16)$ \\
\hline $1-<4$ & 5,751 & 2,437 & 2.00 & 0.77 & $(0.66,0.90)$ & 0.83 & $(0.69,0.99)$ & 0.85 & $(0.71,1.01)$ & 0.84 & $(0.72,0.98)$ & 0.93 & $(0.81,1.05)$ \\
\hline$\geq 4$ & 3,729 & 1,518 & 6.84 & 0.63 & $(0.50,0.80)$ & 0.68 & $(0.52,0.90)$ & 0.72 & $(0.53,0.96)$ & 0.70 & $(0.54,0.90)$ & 0.84 & $(0.71,1.00)$ \\
\hline$p$-trend & & & & $<0.01$ & & $<0.01$ & & $<0.01$ & & $<0.01$ & & 0.04 & \\
\hline
\end{tabular}

HR and $95 \% \mathrm{Cl}$ were derived from the modified Cox proportional hazard model by age at baseline and are based on pooled estimates from country specific analyses using a random effects meta-analysis.

Model 1: sex, smoking status, physical activity level, and education level.

Model 2: additional to model 1: intake of energy, protein, carbohydrates, saturated fatty acids, mono-unsaturated fatty acids, poly-unsaturated fatty acids, alcohol, and fiber.

Model 3: additional to model 2: intake of coffee, juices, soft-drinks, and milk.

Model 4: additional to model 3: body mass index.

doi:10.1371/journal.pone.0036910.t002 


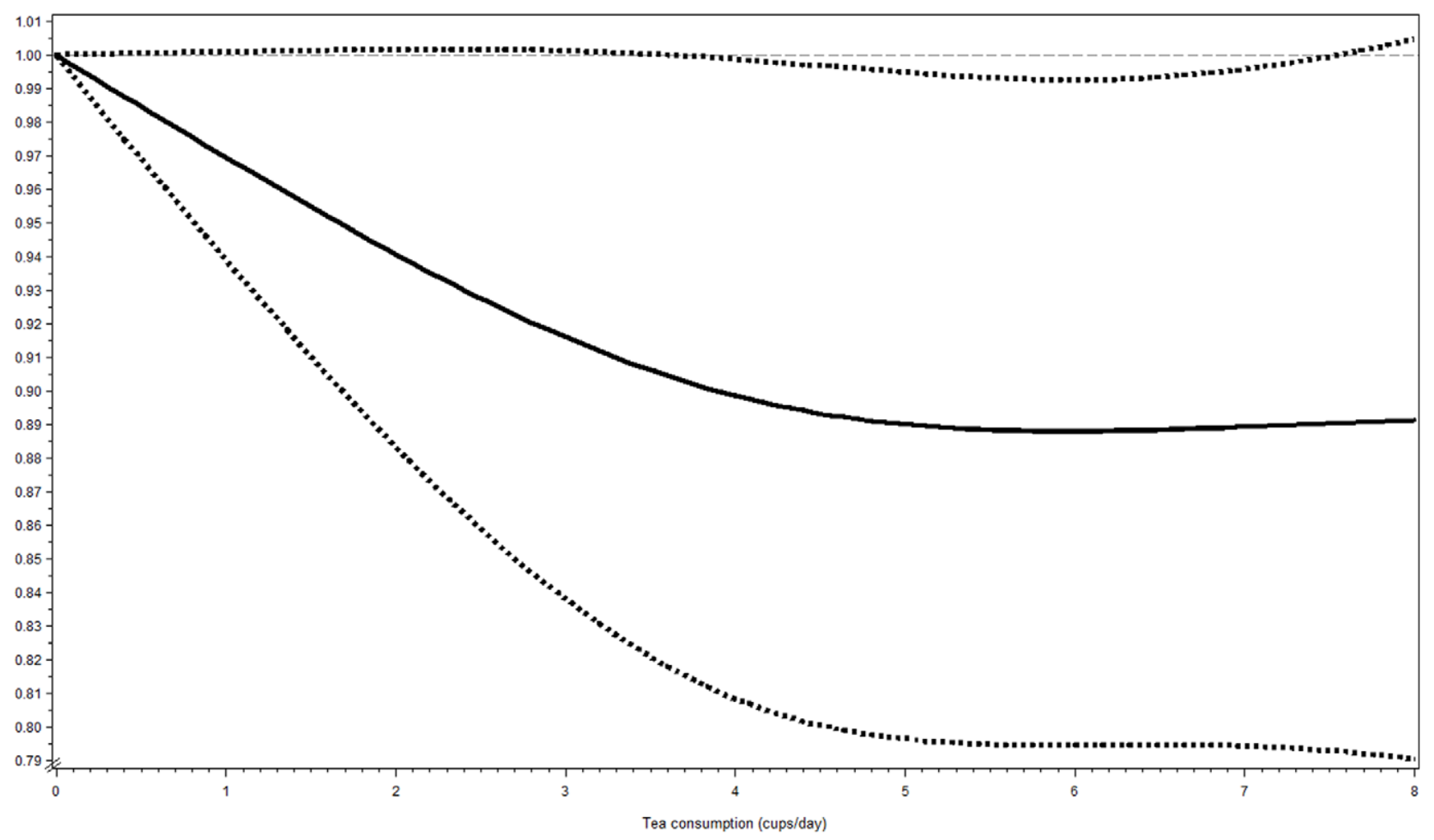

Figure 3. Association between tea consumption based on data from a food frequency questionnaire and risk of type 2 diabetes obtained by spline regression with 3 knots $(1,4,7$ cups per day) and 0 cups per day as reference $(n=26,039)$. Dotted lines represent $95 \%$ confidence intervals $(95 \% \mathrm{Cl}) . P_{\text {non-linearity }}=0.20$. Hazard ratios were adjusted for sex, smoking status, physical activity level, education level, intake of energy, protein, carbohydrates, saturated fatty acids, mono-unsaturated fatty acids, poly-unsaturated fatty acids, alcohol, fiber, coffee, juices, softdrinks, milk, and body mass index.

doi:10.1371/journal.pone.0036910.g003

estimates much. This may indicate that the effect of a healthier lifestyle could not be adequately adjusted for due to measurement error in these factors. Even though inclusion of BMI into the model attenuated the association between tea consumption and risk of type 2 diabetes, residual confounding by BMI might also be present. As BMI could also be considered as intermediate [33], however, caution should be taken when interpreting the model including BMI.

Furthermore, the inclusion of only clinical cases of diabetes rather than undiagnosed cases of diabetes might have limited our results if the prevalence of undiagnosed cases of diabetes differed substantially among tea categories. We have no indication, however, that this differential misclassification in the outcome was likely.

In conclusion, we observed a linear inverse association between tea consumption and incidence of type 2 diabetes. People who drink at least 4 cups of tea per day may have a $16 \%$ lower risk of developing type 2 diabetes than non-tea drinkers. Whether consumption of all types of tea is associated similarly with lower risk of type 2 diabetes and whether this association is causal should be further investigated.

\section{Acknowledgments}

We thank all EPIC participants and staff for their contribution to the study.

The InterAct Consortium list of authors and affiliations is as follows:

Geertruida J van Woudenbergh ${ }^{1}$, Anneleen Kuijsten ${ }^{1}$, Dagmar Drogan $^{2}$, Daphne L van der A ${ }^{3}$, Dora Romaguera ${ }^{4}$, Eva Ardanaz ${ }^{5}$, Pilar Amiano $^{6}$, Aurelio Barricarte ${ }^{5}$, Joline WJ Beulens ${ }^{7}$, Heiner Boeing ${ }^{2}$, H. Bas
Bueno-de-Mesquita ${ }^{3}$, Christina C Dahm ${ }^{8}$, M-Doleres Chirlaque ${ }^{9}$, Francoise Clavel $^{10}$, Francesca L Crowe ${ }^{11}$, Piia-Piret Eomois ${ }^{12}$, Guy Fagherazzi $^{10}$, Paul W Franks ${ }^{13}$, Jytte Halkjær ${ }^{14}$, Kay $\mathrm{T}$ Khaw ${ }^{15}$, Giovanna Masala $^{16}$, Amalia Mattiello ${ }^{17}$, Peter Nilsson ${ }^{13}$, Kim Overvad ${ }^{8}$, J. Ramón Quirós $^{18}$, Olov Rolandsson ${ }^{19}$, Isabelle Romieu ${ }^{20}$, Carlotta Sacerdote ${ }^{21}$, María-José Sánchez ${ }^{22}$, Matthias B Schulze ${ }^{2}$, Nadia Slimani ${ }^{20}$, Ivonne Sluijs $^{7}$, Annemieke MW Spijkerman ${ }^{3}$, Giovanna Tagliabue ${ }^{23}$, Birgit Teucher $^{12}$, Anne Tjønneland ${ }^{14}$, Rosario Tumino ${ }^{24}$, Nita G Forouhi ${ }^{25}$, Stephen Sharp ${ }^{25}$, Claudia Langenberg ${ }^{25}$, Edith JM Feskens ${ }^{1}$, Elio Riboli ${ }^{4}$, Nicholas J Wareham ${ }^{25}$.

${ }^{1}$ Wageningen University, Division of Human Nutrition, Wageningen, The Netherlands; ${ }^{2}$ German Institute of Human Nutrition PotsdamRehbruecke, Germany; ${ }^{3}$ National Institute for Public Health and the Environment (RIVM), Bilthoven, The Netherlands; ${ }^{4}$ Imperial College London, Department of Epidemiology\&Biostatistics, School of Public Health, London, United Kingdom; ${ }^{5}$ Navarre Public Health Institute, Pamplona, Spain; ${ }^{6}$ Public Health Division of Gipuzkoa, San Sebastian, Spain; ${ }^{7}$ University Medical Center, Utrecht, The Netherlands; ${ }^{8}$ School of Public Health, Aarhus University, Aarhus, Denmark; ${ }^{9}$ Murcia Regional Health Authority, Spain; ${ }^{10}$ Institut National de la Santé et de la Recherche Médicale (INSERM) and Center for Research in Epidemiology and Population Health (CESP), France; ${ }^{11}$ University of Oxford, Oxford, United Kingdom; ${ }^{12}$ German Cancer Research Center, Heidelberg, Germany; ${ }^{13}$ Lund University, Malmö, Sweden; ${ }^{14}$ Danish Cancer Society, Institute of Cancer Epidemiology, Copenhagen, Denmark; ${ }^{15}$ University of Cambridge, Cambridge, United Kingdom; ${ }^{16}$ Cancer Research and Prevention Institute (ISPO), Florence, Italy; ${ }^{17}$ Federico II University, Naples, Italy; ${ }^{18}$ Asturias Health \& Health Care Council, Oviedo, Spain; ${ }^{19}$ Department of Public Health and Clinical Medicine, Family Medicine, Umeå University, Umeå, Sweden; ${ }^{20}$ International Agency for Research of Cancer, Lyon, France; ${ }^{21}$ Center for Cancer Prevention, Torino, Italy; ${ }^{22}$ Andalusian School of Public Health, Granada, Spain; ${ }^{23}$ Cancer Registry and Environmental Epidemiology Unit, Fondazione IRCCS Istituto 
Nazionale dei Tumori, Milan, Italy; ${ }^{24}$ Cancer Registry and Histopathology Unit, Ragusa, Italy; ${ }^{25} \mathrm{MRC}$ Epidemiology Unit, Cambridge, United Kingdom

\section{References}

1. Wild S, Roglic G, Green A, Sicree R, King H (2004) Global prevalence of diabetes: estimates for the year 2000 and projections for 2030. Diabetes Care 27(5): 1047-1053.

2. Abdullah A, Peeters A, de Courten M, Stoelwinder J (2010) The magnitude of association between overweight and obesity and the risk of diabetes: a metaanalysis of prospective cohort studies. Diabetes Res Clin Pract 89(3): 309-319.

3. Manikandan R, Sundaram R, Thiagarajan R, Sivakumar MR, Meiyalagan V, et al. (2009) Effect of black tea on histological and immunohistochemical changes in pancreatic tissues of normal and streptozotocin-induced diabetic mice (Mus musculus). Microsc Res Tech 72(10): 723-726.

4. Lenzen S (2008) Oxidative stress: the vulnerable beta-cell. Biochem Soc Trans 36(Pt 3): 343-347.

5. Kao YH, Chang HH, Lee MJ, Chen CL (2006) Tea, obesity, and diabetes. Mol Nutr Food Res 50(2): 188-210.

6. Jing Y, Han G, Hu Y, Bi Y, Li L, et al. (2009) Tea consumption and risk of type 2 diabetes: a meta-analysis of cohort studies. J Gen Intern Med 24(5): 557-562.

7. Sartorelli DS, Fagherazzi G, Balkau B, Touillaud MS, Boutron-Ruault MC, et al. (2010) Differential effects of coffee on the risk of type 2 diabetes according to meal consumption in a French cohort of women: the E3N/EPIC cohort study. Am J Clin Nutr. 2010; 91(4): 1002-1012.

8. Oba S, Nagata G, Nakamura K, Fujii K, Kawachi T, Takatsuka N, et al. (2010) Consumption of coffee, green tea, oolong tea, black tea, chocolate snacks and the caffeine content in relation to risk of diabetes in Japanese men and women. Br J Nutr 103(3): 453-459.

9. Van Dieren S, Uiterwaal CS, van der Schouw YT, van der AD, Boer JM, et al. (2009) Coffee and tea consumption and risk of type 2 diabetes. Diabetologia 52(12): 2561-2569.

10. Boggs DA, Rosenberg L, Ruiz-Narvaez EA, Palmer JR (2010) Coffee, tea, and alcohol intake in relation to risk of type 2 diabetes in African American women. Am J Clin Nutr 92(4): 960-966.

11. Huxley R, Lee CM, Barzi F, Timmermeister L, Czernichow S, et al. (2009) Coffee, decaffeinated coffee, and tea consumption in relation to incident type 2 diabetes mellitus: A systematic review with meta-analysis. Arch Intern Med 169(22): 2053-2063.

12. Riboli E, Hunt KJ, Slimani N, Ferrari P, Norat T, et al. (2002) European Prospective Investigation into Cancer and Nutrition (EPIC): study populations and data collection. Public Health Nutr 5(6B): 1113-1124.

13. The InterAct Consortium (2011) Design and cohort description of the InterAct Project: an examination of the interaction of genetic and lifestyle factors on the incidence of type 2 diabetes in the EPIC Study. Diabetologia 54(9): 2272-2282.

14. University FaAOWHOUN. Energy and Protein Requirements (1985) Report of a Joint FAO/WHO/UNU Expert Consultation World Health Organization Technical Report Series 724. Geneva, Switzerland: WHO.

15. Wareham NJ, Jakes RW, Rennie KL, Schuit J, Mitchell J, et al. (2003) Validity and repeatability of a simple index derived from the short physical activity questionnaire used in the European Prospective Investigation into Cancer and Nutrition (EPIC) study. Public Health Nutr 6(4): 407-413.

16. Onland-Moret NC, van der AD, van der Schouw YT, Buschers W, Elias SG, et al. (2007) Analysis of case-cohort data: a comparison of different methods. J Clin Epidemiol 60(4): 350-355.

\section{Author Contributions}

Conceived and designed the experiments: The InterAct Consortium. Performed the experiments: The InterAct Consortium. Analyzed the data: GJvW. Contributed reagents/materials/analysis tools: The InterAct Consortium. Wrote the paper: The InterAct Consortium.

17. Higgins JP, Thompson SG (2002) Quantifying heterogeneity in a meta-analysis. Stat Med 21(11): 1539-1558.

18. Fukino Y, Ikeda A, Maruyama K, Aoki N, Okubo T, et al. (2008) Randomized controlled trial for an effect of green tea-extract powder supplementation on glucose abnormalities. Eur J Clin Nutr 62(8): 953-960.

19. Hosoda K, Wang MF, Liao ML, Chuang GK, Iha M, et al. (2003) Antihyperglycemic effect of oolong tea in type 2 diabetes. Diabetes Care 26(6): $1714-1718$.

20. Huyen VT, Phan DV, Thang P, Hoa NK, Ostenson CG (2010) Antidiabetic effect of Gynostemma pentaphyllum tea in randomly assigned type 2 diabetic patients. Horm Metab Res 42(5): 353-357.

21. Nagao T, Meguro S, Hase T, Otsuka K, Komikado M, et al. (2009) A catechinrich beverage improves obesity and blood glucose control in patients with type 2 diabetes. Obesity (Silver Spring). 17(2): 310-317.

22. Ryu OH, Lee J, Lee KW, Kim HY, Seo JA, et al. (2006) Effects of green tea consumption on inflammation, insulin resistance and pulse wave velocity in type 2 diabetes patients. Diabetes Res Clin Pract 71(3): 356-358.

23. Stendell-Hollis NR, Thomson CA, Thompson PA, Bea JW, Cussler EC, et al. (2010) Green tea improves metabolic biomarkers, not weight or body composition: a pilot study in overweight breast cancer survivors. J Hum Nutr Diet 23(6): 590-600.

24. Carlsen MH, Halvorsen BL, Holte K, Bohn SK, Dragland S, et al. (2010) The total antioxidant content of more than 3100 foods, beverages, spices, herbs and supplements used worldwide. Nutr J 9(1): 3.

25. Lakenbrink C, Lapczynski S, Maiwald B, Engelhardt UH (2000) Flavonoids and other polyphenols in consumer brews of tea and other caffeinated beverages. J Agric Food Chem 48(7): 2848-2852.

26. Kyle JA, Morrice PC, McNeill G, Duthie GG (2007) Effects of infusion time and addition of milk on content and absorption of polyphenols from black tea. J Agric Food Chem 55(12): 4889-4894.

27. Arts MJ, Haenen GR, Wilms LC, Beetstra SA, Heijnen GG, et al. (2002) Interactions between flavonoids and proteins: effect on the total antioxidant capacity. J Agric Food Chem 50(5): 1184-1187.

28. Hollman PC, Van Het Hof KH, Tijburg LB, Katan MB (2001) Addition of milk does not affect the absorption of flavonols from tea in man. Free Radic Res 34(3): 297-300.

29. Leenen R, Roodenburg AJ, Tijburg LB, Wiseman SA (2000) A single dose of tea with or without milk increases plasma antioxidant activity in humans. Eur J Clin Nutr 54(1): 87-92.

30. Van het Hof KH, Wiseman SA, Yang CS, Tijburg LB (1999) Plasma and lipoprotein levels of tea catechins following repeated tea consumption. Proc Soc Exp Biol Med 220(4): 203-209.

31. Langley-Evans SC (2000) Consumption of black tea elicits an increase in plasma antioxidant potential in humans. Int J Food Sci Nutr 51(5): 309-315.

32. Serafini M, Ghiselli A, Ferro-Luzzi A (1996) In vivo antioxidant effect of green and black tea in man. Eur J Clin Nutr 50(1): 28-32.

33. Grove KA, Lambert JD (2010) Laboratory, Epidemiological, and Human Intervention Studies Show That Tea (Camellia sinensis) May Be Useful in the Prevention of Obesity. J Nutr 140(3): 446-453. 